\title{
Rheological Characterization of Polyolefin Composites with Reduced Flammability
}

\author{
Veronika Habrova $^{\mathrm{a}}$, Alena Kalendova ${ }^{\mathrm{b}, \mathrm{c}}$ and Gordana Paravanova ${ }^{\mathrm{c}}$ \\ ${ }^{a}$ Polymer Institute Brno, Tkalcovská 36/2 Brno 656 49, Czech Republic \\ ${ }^{b}$ Department of Polymer Engineering, Tomas Bata University in Zlin, TGM 275, Zlin 76272, Czech Republic \\ ${ }^{c}$ Centre of Polymer Systems, University Institute, Tomas Bata University in Zlín, Nad Ovčirnou 3685, 76001 Zlín, \\ Czech Republic
}

\begin{abstract}
In this work, the low-flammability halogen-free polyolefin composites were characterized with three rheological methods. In the extrusion process of the studied materials the end-products with a dissimilar surface quality were produced. Therefore, the diverse melt flow behaviours evaluated with common rheological techniques were also expected. Nevertheless, the conventional rotational and capillary rheometries were not able to describe the differences between investigated flame retarded composites in optimal way. Thus, the non-conventional rheological die Shark skin, originally designed to detect flow instabilities, was tested as a third possibility in order to better understand rheology of the filled polymer melts. It was found that the Shark skin technique is able to characterize the studied two kind flame retardants composites and can also be helpful to qualify the production bathes with identical additives/polymer formulation.
\end{abstract}

Keywords: Rheology, halogen-free flame retardant, polyolefin composite.

PACS: $83.85 . \mathrm{Cg}$, 83.60.Wc, $83.80 . \mathrm{Sg}$

\section{INTRODUCTION}

Significant progress in the development of flame retarded halogen-free polymers has been made and brominated flame retardants (BrFR) have been replaced with halogen-free flame retardants (HFFR) more commonly in recent years. Unfortunately, a majority of commercially available HFFR are less effective than BrFR. It causes a need for a higher loading of active matter and thus considerable influences of the composite properties, particularly the flow behaviour. Not many studies have discussed rheological properties of HFFR composites based on polyethylene $(\mathrm{PE})^{1}$, polypropylene $(\mathrm{PP})^{2}$ and other polymers ${ }^{3,4}$. The measurements have often been carried out by conventional methods like rotational and capillary rheometries. Recently, thermal scanning rotational rheometer has been frequently used to study efficiency mechanism of flame retardants. These measurements revealed for example a strong link between high temperature viscosity and foaming ${ }^{2}$. On the other hand, the studies concerning characterization of flow behaviour as a tool predicting the quality of end-products are rather rare.

The aim of this study is to show how the non-conventional rheological die Shark skin ${ }^{5}$ can be helpful to characterize the filled melts when other techniques fail.

\section{EXPERIMENTAL}

The studied HFFR/PP compounds marked FR1-PP, FR2-PP and HFFR/PE compounds marked FR3-PE, FR4-PE were prepared on laboratory twin-screw extruder, and on a production line equipped with twin-screw extruder used for small-scale masterbatch production, respectively. Both sample groups were compounded in the way suitable for high-filled mixtures. The intumescent flame retardants used in the work are commercially available and act well in polyolefines. ADK STAB FP-2200 (Adeka Palmarole SAS) is a mixture of piperazine pyrophosphate, blend of phosphoric acid and zinc oxide. Exolit AP 766 (Clariant) is a surface modified ammonium polyphosphate. The concentrations HFFR additives were 18 wt.\% for PP compounds and 26 wt.\% for PE compounds. Mosten EH 501,

6th International Conference on Times of Polymers (TOP) and Composites

AIP Conf. Proc. 1459, 268-270 (2012); doi: 10.1063/1.4738465

(C) 2012 American Institute of Physics 978-0-7354-1061-9/\$30.00 
Liten BB 29, Exxomobil LL 6201 XR and Exxomobil LL 6101 served as polymer matrixes. Despite the fact that the formulations and processing conditions of FR3-PE and FR4-PE batches were identical, the same processing behaviour in the final extrusion was not observed likewise in the case of the first group of materials. The end products - micropipes showed a different quality of outer surface which was logically undesirable. Therefore, we decided to study the rheology of these composites in detail. The rheological behaviour of filled melts was firstly measured on Bohlin Rheometer CSM 50 equipped with plate-plate rotational geometry in controlled strain oscillation regime. The deformation was $0.5 \%$. Secondly, the twin-bore capillary rheometer Rosant RH 10 was employed. Zero-length capillary and $16 \mathrm{~mm}$ length as non-zero capillary, both with $1 \mathrm{~mm}$ diameter, were applied. The final testing was performed with the capillary rheometer Goettfert Rheograph 120 equipped with special Shark skin slit die with dimension $0.3 \times 3 \times 30 \mathrm{~mm}$. All measurements were done at $200^{\circ} \mathrm{C}$.

\section{RESULTS}

The data obtained on the rotational rheometer are shown in Figure 1. The remarkable shift to higher viscosities can be seen in case of filled compounds FR1-PP and FR2-PP compared to neat PP. Nevertheless, there is no visible difference between the two kinds of flame retardant fillings. As for the bathes FR3-PE and FR4-PE with equal formulations, the curve courses were not fully identical but similar, with a shift to higher viscosities for FR3-PE.

The flow curves measured with the help of capillary rheometry are depicted on the Figure 2. Although small differences between the samples FR3-PE and FR4-PE were found in shear flow dependences, in the case of extensional flow only neat PP showed diverse course of the flow curve. These data are surprisingly in the contrast to a general experience that the extensional viscosity is in most cases more sensitive to difference in materials.

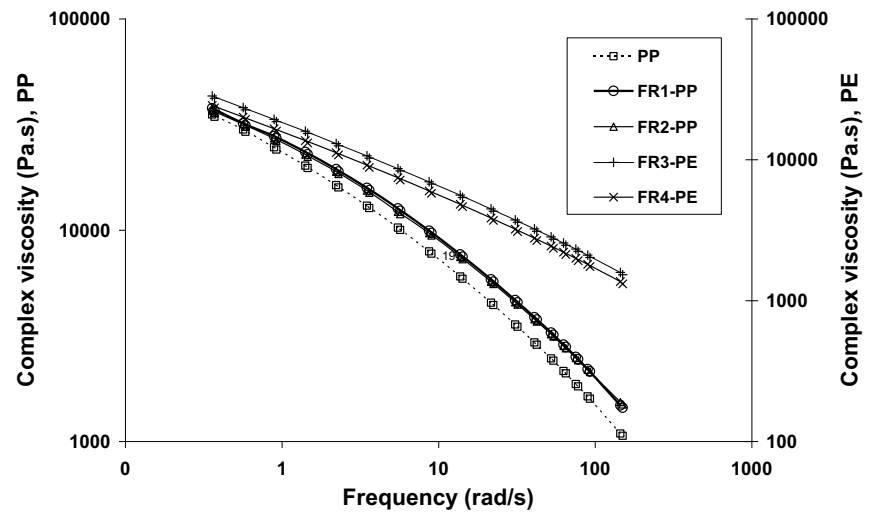

FIGURE 1. Flow behaviour of polyolefin/HFFR composites: rotational rheometry.

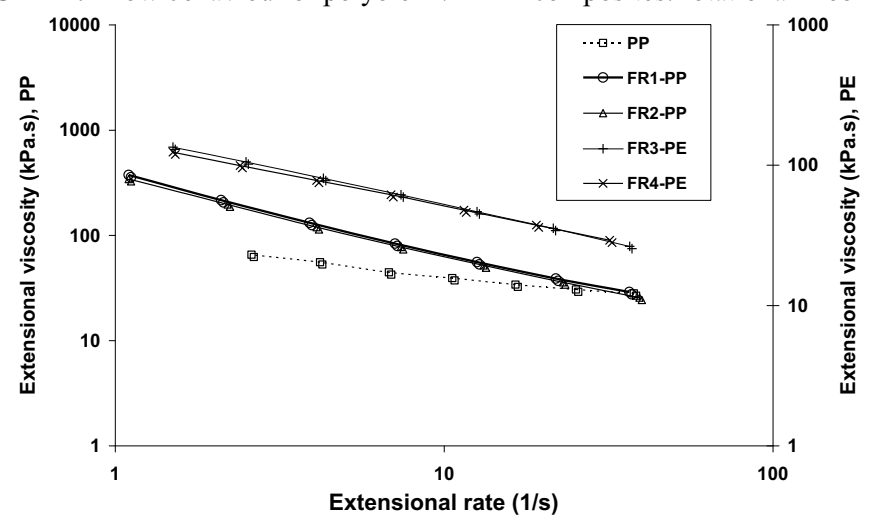

FIGURE 2. Flow behaviour of polyolefin/HFFR composites: capillary rheometry.

The Figure 3 shows the example of pressure signal gained from Shark skin die measurement and plotted as an amplitude of the pressure deviation in the frequency scale. The analogous data were generated at several shear rates from 10 to $5000 \mathrm{~s}^{-1}$ for all studied samples. Significant samples differences that indicated flow instabilities were detected at shear rate of $50 \mathrm{~s}^{-1}$ for FR1-PP and FR2-PP (see Fig. 3) and at shear rates of 2000 and $5000 \mathrm{~s}^{-1}$ for 
FR3-PE and FR4-PE samples. On the assumption that the flow instability shark skin is responsible for poor-quality of final products, the third pressure transducer signal was particularly assessed from the point of view of pressure amplitude deviation. As for the first sample group, the initial flow instabilities were noticed for FR2-PP (see the arrow in the picture). The material FR2-PP was considered as a poorer-quality one during final processing coincidently. In the case of the second sample group, the situation was more complicated. The initial significant pressure amplitude deviations were observed at shear rate of $2000 \mathrm{~s}^{-1}$ for the batch FR4-PE. Nevertheless, the deviations did not reach such high magnitudes. On the other hand, a much more intensive pressure increase was detected at shear rate of $5000 \mathrm{~s}^{-1}$ for the batch FR3-PE which was evaluated as inferior during processing. We assume that the minor flow instabilities probably do not cause the quality problems of final product, and the surface roughness of the products may rather occurs in the case of the bigger pressure deviations come.
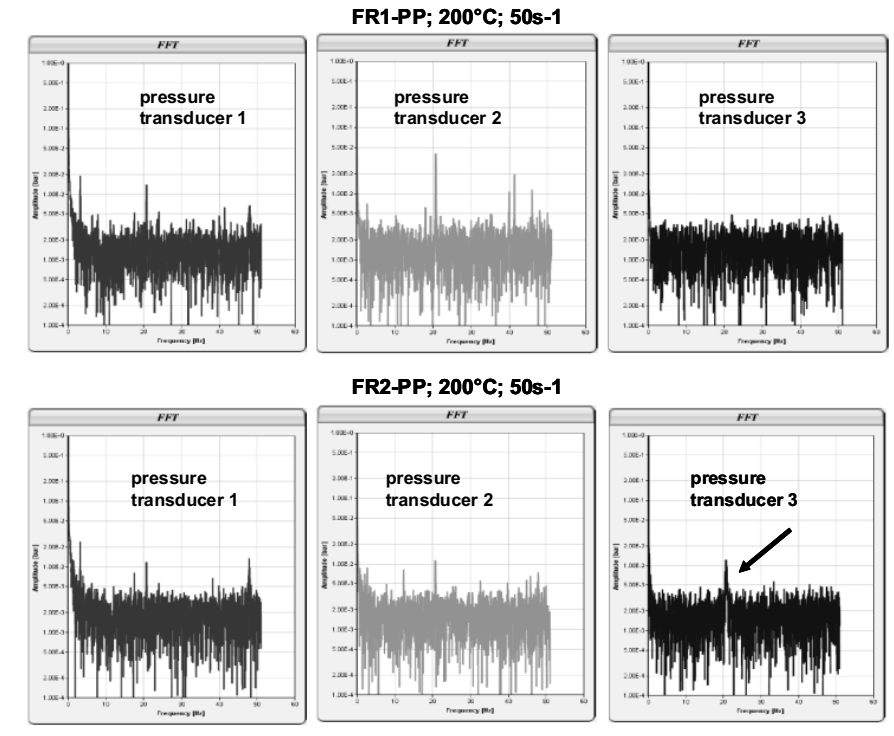

FIGURE 3. Graphs from "Shark skin" die measurements: the arrow shows occurrence of flow instabilities.

\section{CONCLUSIONS}

Three rheological techniques were applied to characterize flow behaviour of polyolefin/HFFR melts. The rotational and capillary rheometries were not fully sufficient for a precise description of the composites, significantly varied from final-processing point of view. On the other hand, the rheological die Shark skin was able to distinguish the studied materials and recognize the occurrence of flow instabilities. The results well corresponded to end-production responses of the assessed materials.

\section{ACKNOWLEDGMENTS}

This article was written with support of the project FR-TI1/188 granted by Ministry of Industry and Trade of Czech Republic, and Operational Program Research and Development for Innovations co-funded by the European Regional Development Fund (ERDF) and national budget of Czech Republic, within the framework of project Centre of Polymer Systems (reg. number: CZ.1.05/2.1.00/03.0111). Special acknowledgement is dedicated to Joachim Sunder, Goettfert GmbH who carried out the Shark skin measurements.

\section{REFERENCES}

1. Z. Wang, Z. Chen and W. Fan, Polymer-Plastics Technology Engineering, 45, 191-196 (2006).

2. P. Anna, G. Marosi, S. Bourbigot, M. Le Bras and R. Delobel, Polymer Stability and Degradation, 77, $243-247$ (2002).

3. L. Di Maio, D. Acierno and D. Di Martino, Macromol. Symp. , 247, 371-378 (2007).

4. M. Choi, Y. Kim, J. Kim and H.Kim, Korea-Australia Rhelogy Jounal , 20, 4, 245-251 (2008).

5. J .Sunder, "Fourier Transformation Analysis in Capillary Flow-A New Option to Detect Flow Instabilities (Shark Skin)" in Novel Trend in Rheology III, edited by Martin Zatloukal, AIP Conference Proceedings 1152, 2009, pp. 152-160. 
Copyright of AIP Conference Proceedings is the property of American Institute of Physics and its content may not be copied or emailed to multiple sites or posted to a listserv without the copyright holder's express written permission. However, users may print, download, or email articles for individual use. 